\title{
PROPAGACIÓN VEGETATIVA DE FERNANSÁNCHEZ (Triplaris guayaquilensis) MEDIANTE LA UTILIZACIÓN DE HORMONAS DE ENRAIZAMIENTO (ANA Y AIB)
}

\author{
Nicolás Cruz Rosero ${ }^{1}$, Jaime Morante Carriel $^{1}$ y Maura Acosta Anzules ${ }^{3}$ \\ ${ }^{1}$ Laboratorio de Biotecnología. Unidad de Investigación Científica y Tecnológica, Universidad Técnica Estatal de Quevedo \\ Km 1 11/2 via Quevedo a Santo Domingo de los Colorados, casilla 73 Quevedo, Los Ríos, Ecuador. nicolascruz83@gmail.com \\ ${ }^{3}$ Facultad de Ciencias Ambientales, Universidad Técnica Estatal de Quevedo. Quevedo, Los Ríos, Ecuador
}

\section{RESUMEN}

La presente investigación se la realizó en el Laboratorio de Biotecnología de la Universidad Técnica Estatal de Quevedo, los objetivos planteados fueron establecer una metodología para la propagación vegetativa de Fernansánchez (Triplaris guayaquilensis) con el uso de hormonas de enraizamiento (ANA y AIB), y determinar las mejores concentraciones de hormonas de ANA y AIB en el enraizamiento de ramillas de esta especie. Se aplicó un diseño completamente al azar con 5 tratamientos, 3 repeticiones y diez unidades experimentales por repeticiones. A los 45 días, los mejores tratamientos para el enraizamiento del Fernansánchez con (ANA y AIB) se obtuvieron en las concentraciones $1000 \mathrm{mg} \mathrm{kg} \mathrm{k}^{-1} \mathrm{ANA}+1000 \mathrm{mg} \mathrm{kg}^{-1} \mathrm{AIB}$ para la longitud promedio de la raíz mayor con $17.67 \mathrm{~cm} ; 500 \mathrm{mg} \mathrm{kg}^{-1} \mathrm{ANA}+500 \mathrm{mg} \mathrm{kg}^{-1} \mathrm{AIB}$, para el número promedio de raíces y sobrevivencia con 5 raíces y $93.33 \%$ y la concentración de $1000 \mathrm{mg} \mathrm{kg}^{-1} \mathrm{ANA}+1000 \mathrm{mg} \mathrm{kg}^{-1}$ AIB fue ideal para vigor con 2.89 , equivalente a 3 (vigor alto). Con esta investigación queda demostrado que las auxinas ANA y AIB inducen y mejoran la calidad de raíces sobre las estacas de Fernansánchez.

Palabras clave: ANA, AIB, propagación vegetativa, yemas axilares

\begin{abstract}
The present research was made out in Biotechnology Laboratory of Universidad Técnica Estatal ofQuevedo, the objective was to establish a vegetative propagation methodology for Triplaris guayaquilensis (Fernansánchez) with the rooting hormone use (ANA and AIB), as well as to determine the best treatment with the ANA and AIB hormone concentrations in the small branches rooting for this species. Complete Randomized Design with five experimental treatments in three repetitions and ten units by repetitions was used. At 45 days length by the greater root, number of roots, survival, and vigor was evaluated. The best treatments for Fernansánchez with (ANA and AIB) rooting was obtained with $1000 \mathrm{mg} \mathrm{kg}^{-1} \mathrm{ANA}+1000 \mathrm{mg} \mathrm{kg}$-1 AIB concentrations for the length of greater root with $17.67 \mathrm{~cm}$ average, for the number of roots and survival, ideal concentration was $500 \mathrm{mg} \mathrm{kg}^{-1}$ ANA $+500 \mathrm{mg} \mathrm{kg}{ }^{-1}$ AIB with five roots and $93.33 \%$ average and $1000 \mathrm{mg} \mathrm{kg}^{-1} \mathrm{ANA}^{+1000 ~ m g ~ k g}{ }^{-1} \mathrm{AIB}$ concentration was ideal for vigor with 2.89 , equivalent to 3 (high vigor). With this investigation it was demonstrated that the auxin ANA and AIB induce and improve the quality by roots on stakes, taking in consideration that these results cannot be generalized for other species.
\end{abstract}

Key words: ANA, AIB, vegetative propagation, axillary yolks

\section{INTRODUCCIÓN}

La propagación clonal o asexual de especies forestales es una herramienta que usa partes vegetativas para multiplicarla. La propagación vegetativa comprende desde procedimientos sencillos, hasta procedimientos tecnológicamente muy avanzados, basados en la tecnología del cultivo de tejidos genéticamente homogénea, mejorada y libre de plagas y enfermedades. La utilización de tejidos vegetales permite conservar la potencialidad del material seleccionado (Zobel y Talbert, 1988).

El Fernansánchez (Triplaris guayaquilensis) se encuentra distribuido ampliamente en el bosque húmedo tropical, en lugares totalmente secos. Es un árbol de

Recibido: Mayo, 2007. Aceptado: Noviembre: 2007. Publicado como ARTÍCULO en Ciencia y Tecnología 1: 7-10. 2008. mediano tamaño que crece alrededor de $50 \mathrm{~cm}$ de DAP y $25 \mathrm{~m}$ de altura, y de reproducción sexual. En el Ecuador esta especie está distribuida desde la provincia de Esmeraldas hasta Los Ríos, Guayas, El Oro y Loja (Little y Dixón, 1987). Es una especie que se encuentra amenazada por el ritmo acelerado de la deforestación y va disminuyendo constantemente, sin embargo, aún se lo encuentra en huertas de cacao a manera de sombra y en linderos. El establecimiento en plantaciones de Fernansánchez por medio de estacas puede ser una alternativa beneficiosa para desarrollar individuos con mejores características genéticas (Zobel y Talbert, 1988), como fuste recto, que se la utiliza en mueblería por su vistoso color que presenta su madera y estructuras para techo. El objetivo de este trabajo de investigación 
fue el de propagar vegetativamente el Fernansánchez (T. guayaquilensis) con la aplicación de ácido naftalenacético (ANA) y acido Indolbutírico (AIB) estimuladores de enraizamiento.

\section{MATERIALES Y MÉTODOS}

La presente investigación se la realizó en el Laboratorio de Biotecnología de la Universidad Técnica Estatal de Quevedo (UTEQ). El material vegetativo se obtuvo de los viveros de la Finca Experimental "La Represa", propiedad de la UTEQ, localizada a $7.5 \mathrm{~km}$ Vía Quevedo - San Carlos, el sitio de la investigación tiene una altitud de $73 \mathrm{msnm}$, y está situado $79^{\circ} 25^{\prime} 24^{\prime \prime}$ de Longitud Occidental y $1^{\circ} 03$ ' $18^{\prime \prime}$ de Latitud Sur. La temperatura promedio anual es $24.6^{\circ} \mathrm{C}$, precipitación promedio anual de $2,026 \mathrm{~mm}$, heliofanía de $848.2 \mathrm{~h}$ $\mathrm{año}^{-1}$ y humedad relativa $86.0 \%$ (UNIAGRO 2003). El proceso de enraizamiento se lo realizó en el invernadero del laboratorio de la UTEQ.

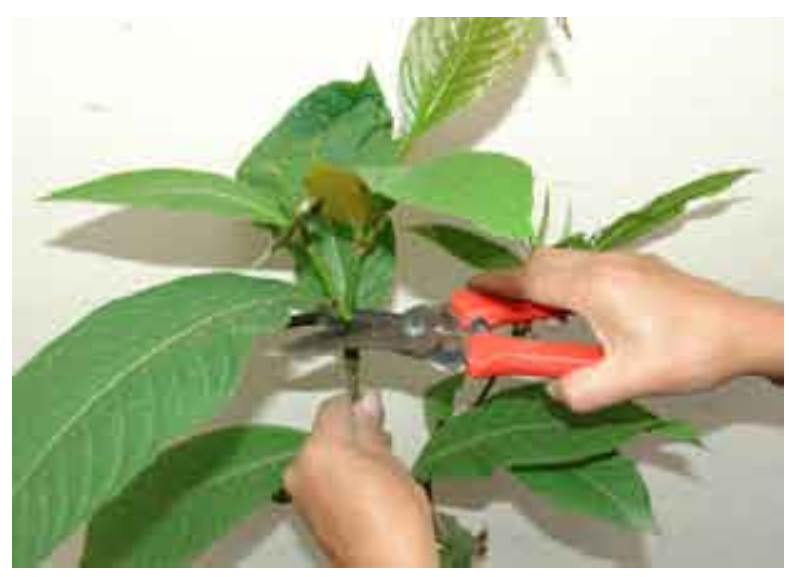

Foto 1. Planta donadora de material vegetal

El material vegetativo para el enraizamiento se lo obtuvo del vivero de plantas de seis meses de edad, se seleccinó dicho material vegetativo, los mismos que se transportaron en recipientes con agua, cada hora se humedecía el follaje para que no pierdan turgencia. Se seleccionaron estacas de $15 \mathrm{~cm}$ de longitud y $0.5 \mathrm{~cm}$ de grosor.

La preparación del sustrato utilizado fue arena y se desinfectó con el fungicida Cobox $1 \mathrm{~g} \mathrm{~L}^{-1}$ una semana antes de la siembra de las estacas. La desinfección del material vegetativo fue sumergir por 15 minutos en una solución del fungicida Cobox con una concentración de 1 $\mathrm{g} \mathrm{L}^{-1}$. Luego se realizó la siembra una vez que las estacas fueran impregnadas en la base de las ramillas con las hormonas ANA y AIB, en diferentes concentraciones. La siembra del material vegetativo se la realizó en bandejas germinadoras, bajo un túnel de polietileno, en un umbráculo cubierto con zarán que permitió el paso del $25 \%$ de luz solar, para evitar el estrés en las estacas (deshidratación). Además, se aplicó riego con una frecuencia de 4 veces al día con un intervalo de 2.5 horas, utilizando un atomizador de 1 litro. A continuación se detallan los tratamientos en estudio: $\mathrm{T}_{0}$ (Sin hormonas), $\mathrm{T}_{1}\left(500 \mathrm{mg} \mathrm{kg}^{-1}\right.$ ANA $+500 \mathrm{mg} \mathrm{kg}^{-1}$ AIB), $\mathrm{T}_{2}$ (1000 mg $\left.\mathrm{kg}^{-1} \mathrm{ANA}+1000 \mathrm{mg} \mathrm{kg}^{-1} \mathrm{AIB}\right), \mathrm{T}_{3}\left(1500 \mathrm{mg} \mathrm{kg}^{-1} \mathrm{ANA}\right.$ $\left.+1500 \mathrm{mg} \mathrm{kg}^{-1} \mathrm{AIB}\right), \mathrm{T}_{4}\left(2000 \mathrm{mg} \mathrm{kg}{ }^{-1}\right.$ ANA +2000 $\mathrm{mg} \mathrm{kg}{ }^{-1} \mathrm{AIB}$ ). En este trabajo se dispuso de un diseño completamente al azar, con cinco tratamientos, tres repeticiones y diez unidades experimentales. A los 45 días se evaluó el porcentaje de enraizamiento, número de raíces, longitud de raíz mayor, supervivencia y vigor

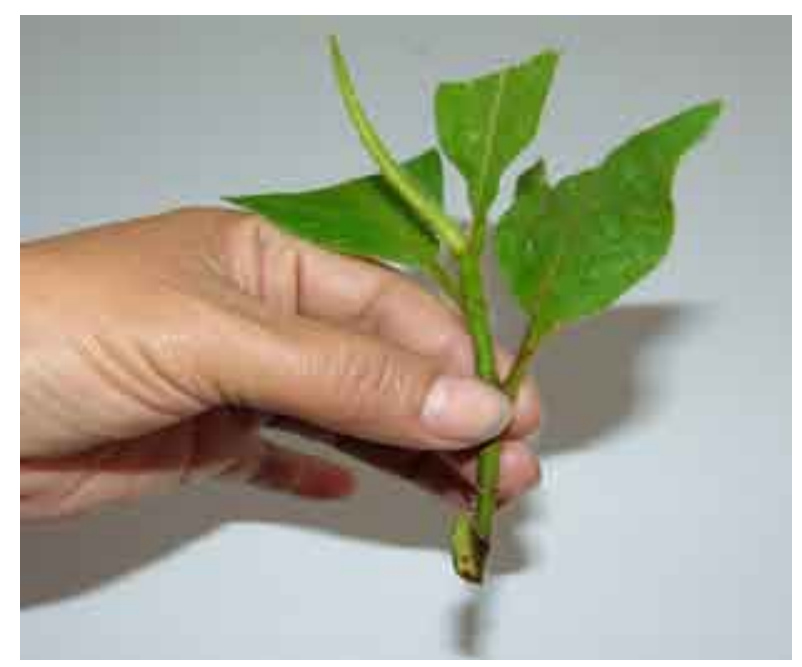

Foto 2. Ramilla ideal para propagación de Fernansánchez

\section{RESULTADOS Y DISCUSIÓN}

\section{Longitud de raíz mayor}

La longitud de raíz presentó diferencias altamente significativas entre los tratamientos en estudio. El tratamiento que mejor respuesta presentó fue la concentración de $1000 \mathrm{mg} \mathrm{kg}^{-1}$ ANA $+1000 \mathrm{mg} \mathrm{kg}^{-1}$ AIB con $17.67 \mathrm{~cm}$ de longitud promedio (Cuadro 1). Así queda demostrado que esta es la concentración favorable para obtener una mayor longitud de raíces. En esta investigación se obtuvo valores que superan a otras investigaciones realizadas utilizando polvos enraizadores, como es el caso de Ramos (2000) en teca quien obtuvo $3.82 \mathrm{~cm}$ de longitud promedio de raíces, evaluado a los 21 días en $1000 \mathrm{mg} \mathrm{kg}^{-1} \mathrm{ANA}+1000 \mathrm{mg}$ $\mathrm{kg}^{-1}$ AIB; Villacís (2003) en moral fino con $3.89 \mathrm{~cm}$ de longitud promedio a los 30 días; Bermúdez (2005) en Gmelina con $6.61 \mathrm{~cm}$ de longitud promedio evaluados a los 2 meses con la misma concentración y Chicaiza 
(2004) de teca con $11.10 \mathrm{~cm}$ de longitud promedio evaluados a los 28 días en el tratamiento de $1500 \mathrm{mg}$ $\mathrm{kg}^{-1}$ ANA $+1500 \mathrm{mg} \mathrm{kg}^{-1}$ AIB.

Cuadro 1. Longitud de la raíz mayor (cm) en la propagación vegetativa de $T$. guayaquilensis con el uso de hormonas de enraizamiento (ANA Y AIB)

\begin{tabular}{lc}
\hline \multicolumn{1}{c}{ Tratamientos } & $\begin{array}{c}\text { Long. raíz } \\
\text { mayor }(\mathbf{c m})\end{array}$ \\
\hline Sin hormonas & $10.70 \mathrm{~b}^{\dagger}$ \\
$500 \mathrm{mg} \mathrm{kg}{ }^{-1} \mathrm{ANA}+500 \mathrm{mg} \mathrm{kg}^{-1} \mathrm{AIB}$ & $11.67 \mathrm{~b}$ \\
$1000 \mathrm{mg} \mathrm{kg}^{-1} \mathrm{ANA}+1000 \mathrm{mg} \mathrm{kg}^{-1} \mathrm{AIB}$ & $17.67 \mathrm{a}$ \\
$1500 \mathrm{mg} \mathrm{kg}^{-1} \mathrm{ANA}+1500 \mathrm{mg} \mathrm{kg}^{-1} \mathrm{AIB}$ & $11.20 \mathrm{~b}$ \\
$2000 \mathrm{mg} \mathrm{kg}^{-1} \mathrm{ANA}+2000 \mathrm{mg} \mathrm{kg}^{-1} \mathrm{AIB}$ & $10.97 \mathrm{~b}$ \\
\hline $\mathbf{C V ~ ( \% )}$ & 5.08 \\
\hline 'Promedios con letras iguales no difieren estadísticamente entre sí, & \\
según a prueba de Duncan $<<0.05$. &
\end{tabular}

También podemos observar que el testigo presentó la menor respuesta, y concuerda con Ramos (2000), cuyo testigo obtuvo la menor longitud de raíz, pero difiere de Hartmann et al. (1990), quienes no utilizaron sustancias promotoras del enraizamiento y obtuvieron buenos resultados. Esto se complementa con lo mencionado por Álvarez y Varona (1988), quienes indican que algunas especies no requieren, pero otras si necesitan ser estimuladas con sustancias auxinitas.

\section{Número de raíces}

La variable número de las raíces reportó diferencias altamente significativas, teniendo al mejor tratamiento a $500 \mathrm{mg} \mathrm{kg}^{-1}$ ANA $+5000 \mathrm{mg} \mathrm{kg}^{-1}$ AIB con promedio de 5.00 raíces (Cuadro 2). Estos resultados superan a los obtenidos por Ramos (2000) donde obtuvo un promedio de 2 raíces con el tratamiento de $1000 \mathrm{mg} \mathrm{kg}^{-1} \mathrm{ANA}+$ $1000 \mathrm{mg} \mathrm{kg}^{-1} \mathrm{AIB}$ en el comportamiento de la teca.

Cuadro 2. Número de raíces en la propagación vegetativa de T. guayaquilensis con el uso de hormonas de enraizamiento (ANA Y AIB)

\begin{tabular}{lc}
\hline \multicolumn{1}{c}{ Tratamientos } & No. Raíces \\
\hline Sin hormonas & $1.53 \mathrm{~b}^{\dagger}$ \\
$500 \mathrm{mg} \mathrm{kg}$ \\
$1000 \mathrm{mg} \mathrm{kg}^{-1} \mathrm{ANA}+500 \mathrm{mg} \mathrm{kg}{ }^{-1} \mathrm{AIB}$ & $5.00 \mathrm{a}$ \\
$1500 \mathrm{mg} \mathrm{kg}^{-1} \mathrm{ANA}+1500 \mathrm{mg} \mathrm{kg}^{-1} \mathrm{AIB}$ & $1.77 \mathrm{~b}$ \\
$2000 \mathrm{mg} \mathrm{kg}^{-1} \mathrm{ANA}+2000 \mathrm{mg} \mathrm{kg}^{-1} \mathrm{AIB}$ & $1.67 \mathrm{~b}$ \\
\hline $\mathbf{C V ~ ( \% )}$ & $1.20 \mathrm{~b}$ \\
\hline
\end{tabular}

Promedios con letras iguales no difieren estadísticamente entre sí, según a prueba de Duncan $\mathrm{p}<0.05$.
Es importante mencionar que a medida que se aumenta las concentraciones de auxinas en los tratamientos en estudio, disminuye el número de raíz. Esto indica que a concentraciones altas de estas hormonas inhiben el desarrollo de raíces, lo que concuerda con Gupta et al. (1980), quienes demostraron que con concentraciones altas se mueren los explantes y que a bajas concentraciones hay un buen desarrollo de las plantas.

\section{Sobrevivencia}

La variable sobrevivencia presentó diferencias altamente significativas, quedando establecido que el tratamiento con mejores resultados fue el de $500 \mathrm{mg}$ $\mathrm{kg}^{-1}$ ANA $+500 \mathrm{mg} \mathrm{kg}^{-1}$ AIB con $93.33 \%$ (Cuadro 3) de sobrevivencia promedio. En esta variable al utilizar diferentes concentraciones de auxinas también existe la misma respuesta que en la variable anterior, a medida que se aumenta la concentración hormonal disminuye la sobrevivencia y la concentración óptima es $500 \mathrm{mg}$ $\mathrm{kg}^{-1}$ ANA $+500 \mathrm{mg} \mathrm{kg}^{-1}$ AIB. Por lo que se infiere que el Fernansánchez debe ser propagado a concentraciones bajas de auxinas.

La respuesta de las estacas al enraizamiento sí fue influenciada por la presencia de las auxinas en los polvos, ya que casi la totalidad de las plantas enraizaron (93.33\%), y concuerda con Valarezo (1984), quien manifestó que las sustancias más utilizadas para estimular el crecimiento radicular son el ácido Indolbutírico y acido naftalenoacético, que son compuestos orgánicos sintetizados en una parte de la planta y que se transloca a otra parte donde a muy bajas concentraciones induce una respuesta fisiológica (Salisbury y Ross 1994). Si se revisan todas las variables antes mencionadas en este estudio, sucede lo mismo, que a mayor concentración de los reguladores del crecimiento vegetal disminuyen las medias en la propagación vegetativa del fernansánchez.

Cuadro 3. Sobrevivencia de las plantas en la propagación vegetativa de $T$. guayaquilensis con el uso de hormonas de enraizamiento (ANA Y AIB)

\begin{tabular}{lc}
\hline \multicolumn{1}{c}{ Tratamientos } & $\begin{array}{c}\text { Sobrevivencia } \\
(\%)\end{array}$ \\
\hline Sin hormonas & $50.00 \mathrm{c}^{\dagger}$ \\
$500 \mathrm{mg} \mathrm{kg}^{-1} \mathrm{ANA}+500 \mathrm{mg} \mathrm{kg} \mathrm{gIB}^{-1} \mathrm{AB}$ & $93.33 \mathrm{a}$ \\
$1000 \mathrm{mg} \mathrm{kg}^{-1} \mathrm{ANA}+1000 \mathrm{mg} \mathrm{kg}^{-1} \mathrm{AIB}$ & $66.70 \mathrm{~b}$ \\
$1500 \mathrm{mg} \mathrm{kg}^{-1} \mathrm{ANA}+1500 \mathrm{mg} \mathrm{kg}^{-1} \mathrm{AIB}$ & $56.70 \mathrm{bc}$ \\
$2000 \mathrm{mg} \mathrm{kg}^{-1} \mathrm{ANA}+2000 \mathrm{mg} \mathrm{kg}^{-1} \mathrm{AIB}$ & $33.30 \mathrm{~d}$ \\
\hline $\mathbf{C V ~ ( \% )}$ & 11.39 \\
\hline
\end{tabular}

Promedios con letras iguales no difieren estadísticamente entre sí, según a prueba de Duncan $\mathrm{p}<0.05$. 


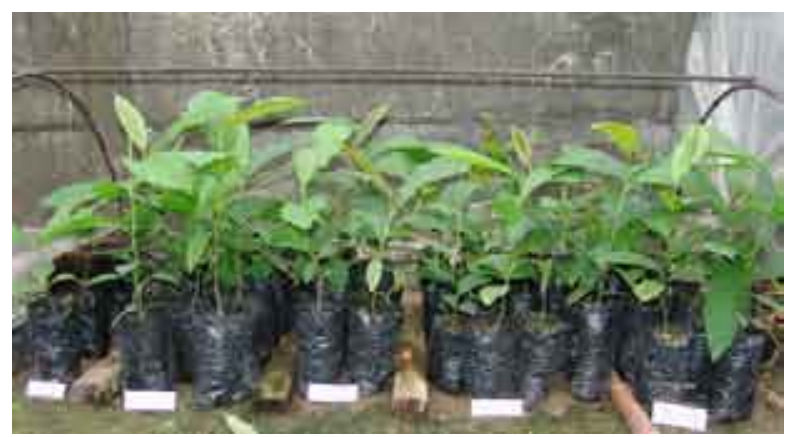

Foto 3. Plantas enraizadas y aclimatadas de Fernansánchez

Vigor

En la variable vigor de plantas se encontraron diferencias altamente significativas, quedando establecidos que el tratamiento con mejores resultados fue el de $1000 \mathrm{mg} \mathrm{kg}^{-1}$ ANA + $1000 \mathrm{mg} \mathrm{kg}^{-1}$ con 2.89 de vigor, aproximado a una categoría 3 (vigor alto). El tratamiento sin hormona reportó un vigor de plantas equivalentes a una categoría baja siendo el tratamiento que presentó el menor promedio (Cuadro 4).

Cuadro 4. Vigor de las plantas en la propagación vegetativa de T. guayaquilensis con el uso de hormonas de enraizamiento (ANA Y AIB)

\begin{tabular}{lc}
\hline \multicolumn{1}{c}{ Tratamientos } & Vigor $^{1 /}$ \\
\hline Sin hormonas & $1.22 \mathrm{c}^{\dagger}$ \\
$500 \mathrm{mg} \mathrm{kg}^{-1} \mathrm{ANA}+500 \mathrm{mg} \mathrm{kg}{ }^{-1} \mathrm{AIB}$ & $1.89 \mathrm{~b}$ \\
$1000 \mathrm{mg} \mathrm{kg}^{-1} \mathrm{ANA}+1000 \mathrm{mg} \mathrm{kg}^{-1} \mathrm{AIB}$ & $2.89 \mathrm{a}$ \\
$1500 \mathrm{mg} \mathrm{kg}^{-1} \mathrm{ANA}+1500 \mathrm{mg} \mathrm{kg}^{-1} \mathrm{AIB}$ & $1.78 \mathrm{~b}$ \\
$2000 \mathrm{mg} \mathrm{kg}^{-1} \mathrm{ANA}+2000 \mathrm{mg} \mathrm{kg}^{-1} \mathrm{AIB}$ & $1.78 \mathrm{~b}$ \\
\hline $\mathbf{C V ~ ( \% )}$ & 9.98 \\
\hline
\end{tabular}

'Promedios con letras iguales no difieren estadísticamente entre sí, según a prueba de Duncan $\mathrm{p}<0.05$

1'Categoría: $1=$ baja; 2 =medio; $3=$ alto

\section{CONCLUSIONES}

Se logró establecer una metodología para la propagación vegetativa de fernansánchez a partir de ramillas, utilizando polvos enraizadores. El mayor número de raíces y porcentaje de sobrevivencia, se presentó con la concentración $500 \mathrm{mg} \mathrm{kg}^{-1}$ ANA +500 mg kg-1 AIB con 5 raíces y $93.33 \%$, respectivamente. Con la concentración $1000 \mathrm{mg} \mathrm{kg}^{-1} \mathrm{ANA}+1000 \mathrm{mg} \mathrm{kg}^{-1}$ AIB se presentaron los mayores resultados para longitud de raíz y vigor con $17.67 \mathrm{~cm}$ y 2.89 , respectivamente.

\section{LITERATURA CITADA}

Álvarez, P. y Varona, J. 1988. Selvicultura. La Habana. Cuba. Edit. Pueblo y Educación. Pagina 22

Bermúdez, M. 2005. Propagación Vegetativa de Gmelina arbórea Rexb. Con el uso de hormonas de enraizamiento
(ANA y AIB) y establecimiento en el campote parcelas permanentes .Tesis de Ingeniero Forestal, Universidad Técnica Estatal de Quevedo. Quevedo-Ecuador. página 41.

Chicaiza, D. 2004. Propagación vegetativa de Tectona grandis L. (Teca) a través de estacas enraizadas. Tesis Ing. Forestal Universidad Técnica Estatal de Quevedo, paginas $37-44$

Gupta, P.; Nadgir, A.; Mascarenhas, A. y Jagannthan V. 1980. Tissue culture of forest trees: clonal multiplication of Tectona grandis L. (teak) by tissue culture. Plant Science letters. 17: 259-268.

Hartmann, H.; Kester, D. y Davies, F. 1990. Plant propagation: principles and practices. 5 ed., Prentice Hall, Englewood cliffs, New yersey, USA. 647 p.

Little, E. y Dixon, R.1987. Árboles Comunes de la Provincia de Esmeraldas, Roma, FAO. Pagina 535

Ramos, L. 2000 Algunos avances en la morfogénesis de la teca. (Tectona grandis) Tesis para obtener la Maestría en Ciencias. Universidad Ciego de Ávila. Ciego de Ávila. Cuba. Pagina. 55

UNIAGRO. 2003. Universidad Técnica Estatal de Quevedo .pagina 38

Valarezo, R. 1984. Rooting hard to root confieres. Vol 35. página 178.

Villacís, O. 2003. Propagación vegetativa de moral fino (Chlorophora tinctoria) con el uso de hormonas de Enraizamiento. Tesis de grado para la obtención del TITULO DE Ingeniería Forestal., Universidad Técnica Estatal de Quevedo. Quevedo-Ecuador. Paginas 50-65

Salisbury, F. y Ross, C. 1994. Fisiología Vegetal. Versión en Español Grupo Editorial Iberoamérica. México. Pag. 395-451

Zobel, B. y Talbert, J. 1988. Técnica de Mejoramiento Genético de Árboles Forestales México. D. F. Limusa. Pagina 27. 